\title{
El papel de la actividad física y el ejercicio en la obesidad
}

\author{
The role of physical activity and exercise in obesity \\ O papel da atividade física e do exercício na obesidade
}

\author{
Diana Cárdenas ${ }^{1 *}$, Ana Lorena Montealegre Páez ${ }^{1}$, Liliana Ladino ${ }^{1}$
}

Recibido: 1 de julio de 2019. Aceptado para publicación: 5 de agosto de 2019

https://doi.org/10.35454/rncm.v2n2.009

\section{Resumen}

Introducción: en los últimos años la obesidad en niños y adultos ha aumentado. La mejor estrategia para el control de esta enfermedad es el manejo médico-nutricional donde la actividad física tiene un papel importante. Sin embargo, todavía existen controversias sobre su impacto en la pérdida y el control del peso corporal.

Objetivos: presentar evidencia actualizada sobre el papel de la actividad física en la etiología de la obesidad y su utilidad en la pérdida y en el control del peso.

Métodos: se realizó una búsqueda en PubMed y Google Scholar con las palabras obesidad, gasto energético y actividad física. Se procede a abordar en primer lugar el papel del gasto energético de la actividad física en la obesidad, luego se analiza el impacto del ejercicio en la pérdida y el control del peso en adultos y niños, y por último se tratan los aspectos más importantes del músculo como órgano endocrino y su papel en los efectos benéficos del ejercicio.

Resultados: la actividad física constante, en una duración mayor a lo recomendado, contribuye a la pérdida y al mantenimiento de peso. Asimismo, tiene un papel preventivo en enfermedades cardiovasculares, osteoporosis, riesgo de fractura, y cáncer de colon, pulmón, próstata, endometrio y de mama.

Conclusiones: la actividad física tiene efectos benéficos demostrados para la salud, en especial para el control de las enfermedades crónicas, para mantener la pérdida de peso a largo plazo y para preservar la masa corporal magra durante una alimentación con restricción energética.

Palabras clave: obesidad, salud pública, metabolismo energético, ejercicio.

\section{Summary}

Introduction: In recent years obesity has increased in children and adults. The best strategy for the control of this disease is the medical-nutritional approach where physical activity has an important role. However, there are still controversies about its impact on the loss and control of body weight.

Objectives: To present the most current evidence on the role of physical activity in the etiology of obesity and its usefulness in weight loss and control.

Methods: A search was conducted in PubMed and Google Scholar with the words obesity, energy expenditure and physical activity. The role of activity energy expenditure in obesity will be addressed first, then the impact of exercise on weight loss and control in adults and children will be analyzed, and finally we will discuss the most important aspects of muscle as an endocrine organ and its role in the beneficial effects of exercise.

Results: Constant physical activity, in a longer duration than recommended, contributes to weight loss and weight maintenance. It also has a preventive role in cardiovascular diseases, osteoporosis, risk of fracture and cancer of the colon, lung, prostate, endometrium and breast.

Conclusions: Physical activity has proven beneficial effects on health, especially for the control of chronic diseases, to maintain long-term weight loss and to preserve lean body mass during an energy-restricted diet.

Keywords: Obesity; Public health; Energy metabolism; Exercise.

\section{Resumo}

Introdução: nos últimos anos, a obesidade em crianças e adultos aumentou. A melhor estratégia para o controle desta doença é o manejo médico-nutricional, onde a atividade física tem um papel importante. No entanto, ainda existem controvérsias sobre o seu impacto na perda e controle do peso corporal.

Objetivos: apresentar evidências atualizadas sobre o papel da atividade física na etiologia da obesidade e sua utilidade na perda e controle de peso.

Métodos: pesquisa realizada no PubMed e Google Scholar com as palavras obesidade, gasto energético e atividade física. O papel do gasto energético da atividade física na obesidade é abordado primeiro, em seguida, é analisado o impacto do exercício na perda de peso e controle em adultos e crianças, e finalmente, os aspetos mais importantes do músculo como órgão endócrino e o seu papel nos efeitos benéficos do exercício.

Resultados: a atividade física constante, em maior tempo do que o recomendado, contribui para a manutenção e perda de peso. Também tem um papel preventivo nas doenças cardiovasculares, na osteoporose, no risco de fraturas e no câncer de cólon, pulmão, próstata, endométrio e mama.

Conclusões: a atividade física tem provado efeitos benéficos na saúde, especialmente para o controle de doenças crônicas, para manter a perda de peso a longo prazo e preservar a massa corporal magra durante uma dieta com restrição de energia.

Palavras-chave: obesidade, saúde pública, metabolismo energético, exercício.
Instituto de Investigación en Nutrición, Genética y Metabolismo, Facultad de Medicina, Universidad El Bosque, Bogotá, D.C., Colombia. 


\section{INTRODUCCIÓN}

El aumento de la prevalencia del sobrepeso y la obesidad en los adultos y los niños es una preocupación importante en la definición de las políticas de salud pública en el mundo. En Colombia, según la Encuesta Nacional de la Situación Nutricional (ENSIN 2015), 37,7 \% de la población adulta tiene sobrepeso y $18,7 \%$ obesidad, siendo esta última más frecuente en las mujeres $(22,4 \%)$ que en los hombres (14,4\%). Esto indica que 56,4 \% de la población adulta colombiana presenta exceso de peso, lo que significa un incremento de 5,2 puntos porcentuales con respecto a 2010. El exceso de peso en los menores en edad escolar se incrementó de 18,8\% en 2010 a 24,4 \% en $2015^{(1)}$. Según la Organización de Naciones Unidas para la Alimentación y Agricultura (FAO) uno de cada cuatro latinoamericanos es obeso con un incremento descontrolado, ya que cada año se suman 3,6 millones de obesos a la región ${ }^{(2)}$. Este es un problema importante de salud pública porque la obesidad tiene efectos negativos en la salud. El riesgo de diabetes tipo 2, de enfermedad cardiovascular, de ciertos tipos de cáncer e incluso la mortalidad son directamente proporcionales al grado de obesidad ${ }^{(3,4)}$. Además, la obesidad tiene consecuencias psicológicas y sociales negativas que impactan la salud en forma directa o indirecta.

Ante la necesidad de plantear un abordaje integral y adecuado, en 1997 la Organización Mundial de la Salud (OMS) definió la obesidad como una enfermedad. Esta enfermedad se caracteriza por el exceso de tejido adiposo (hipertrofia e hiperplasia), resultado de una alimentación desequilibrada donde predomina una ingesta de energía que excede el gasto energético ${ }^{(3)}$. La evolución creciente de la obesidad se explica en parte por un cambio en los estilos de vida, es decir, una creciente sedentarización y un aumento en la disponibilidad de alimentos, siendo esto un reflejo del crecimiento económico mundial. La obesidad es, por lo tanto, una «enfermedad social» con determinantes biológicos (genéticos, hormonales, farmacológicos, metabólicos), conductuales (factores psicológicos y sociales), económicos (papel de la industria agroalimentaria) y ambientales que interactúan entre $\mathbf{s i}^{(s)}$.

La disminución gradual de la actividad física en las sociedades sin duda juega un papel importante en el desarrollo de la obesidad ${ }^{(6)}$. Aproximadamente la mitad de los adultos colombianos realiza 150 minutos semanales de actividad física moderada o 75 minutos semanales de actividad vigorosa o fuerte, como lo reco- mienda la OMS. Cuatro de cada diez mujeres y seis de cada diez hombres cumplen con esta recomendación ${ }^{(1)}$.

Las acciones de salud pública para reducir la obesidad se han centrado principalmente en la promoción de estilos de vida saludables que incluyen una alimentación equilibrada y ejercicio. Sin embargo, este enfoque no ha tenido éxito ya que ningún país ha logrado reducir las tasas de obesidad en los últimos 30 años $^{(7,8)}$. En consecuencia, es pertinente preguntar: ¿Es la incapacidad de restringir la ingesta de energía o de mantener altos niveles de gasto de energía la razón del fracaso? El papel de estos dos elementos aún no se ha determinado de manera concluyente, y su importancia relativa en el manejo del peso ha sido tema de controversia ${ }^{(9-11)}$. Esto ha llevado al cuestionamiento sobre la utilidad de la actividad física en este campo. El objetivo de este artículo es presentar la evidencia actual sobre el papel de la actividad física en la etiología de la obesidad y su utilidad en la pérdida y el control del peso; así como señalar las perspectivas para la investigación en esta área específica. No se pretende realizar una revisión sistemática ni exhaustiva de la literatura. Para ello, se aborda primero el papel del gasto energético, en particular el de la actividad física en la obesidad, luego se analizará el impacto del ejercicio en la pérdida y el control del peso, y por último, los aspectos más importantes del músculo como órgano endocrino y su papel en los efectos benéficos del ejercicio.

\section{¿EXISTE ASOCIACIÓN ENTRE LA OBESIDAD Y LAS ALTERACIONES DEL GASTO ENERGÉTICO?}

El gasto energético total (GET o Total Energy Expenditure) es la cantidad de energía (en calorías) que necesita un organismo para vivir y desarrollar las actividades diarias; está determinado por el tamaño y la composición corporal, por la ingesta de alimentos (termogénesis inducida por los alimentos, TIA) y la actividad física (gasto energético de la actividad física, GEA). El tamaño corporal y la composición corporal son los principales determinantes del gasto energético basal (GEB): a mayor masa libre de grasa mayor necesidad de energía. El gasto energético inducido por la actividad física es el componente más variable del gasto energético total. Por lo tanto, la energía producida por la actividad física es un componente del equilibrio energético particularmente importante en la patogénesis de la obesidad y en su tratamiento ${ }^{(12)}$. Los componentes del gasto energético total se muestran en la Figura 1. 


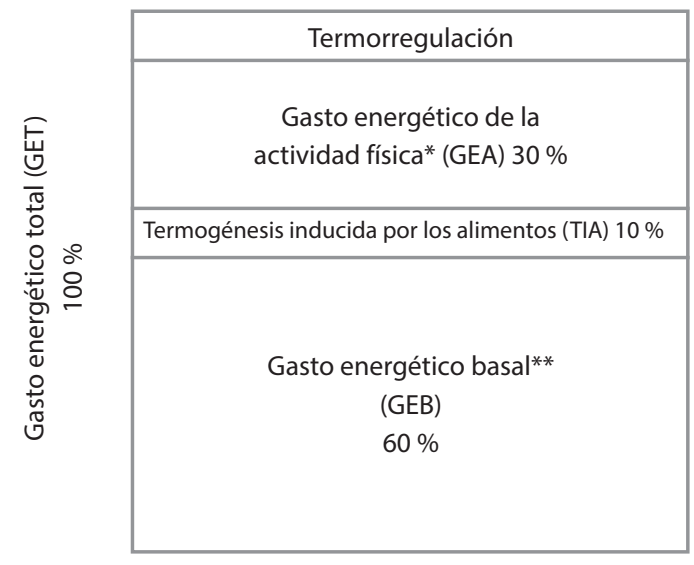

Figura 1. Los componentes del gasto energético total (GET) son el gasto energético basal (GEB), gasto energético de la actividad (GEA) y termogénesis inducida por los alimentos (TIA).* El GEA es el componente más variable del gasto energético total. ${ }^{* *} \mathrm{El}$ GEB se obtiene en condiciones estandarizadas de temperatura, humedad y después de 8 a 12 horas de sueño, ayuno, y en reposo. El gasto energético en reposo (GER) aunque similar no es exactamente el mismo GEB debido a que las condiciones en las que se mide no son las estandarizadas ${ }^{(12)}$.

El tratamiento inicial del sobrepeso y la obesidad incluye cambios en el estilo de vida con una combinación de alimentación equilibrada y ejercicio ${ }^{(13)}$. El objetivo es lograr un balance energético negativo disminuyendo el aporte energético y aumentando el gasto energético total. Una creencia generalizada es que las personas obesas tienen tasas de gasto energético más bajas que las personas no obesas (eutróficas y con sobrepeso), lo que, a largo plazo, podría contribuir a un equilibrio energético positivo y al consecuente aumento de peso. Sin embargo, en una revisión de la literatura de Carneiro et al. donde se evaluaron los estudios que compararon el gasto energético en adultos obesos y no obesos se mostró que, contrario a la creencia popular, las personas obesas tienen un mayor gasto energético basal absoluto y un mayor gasto energético total ${ }^{(14)}$. Cuando se tiene en cuenta la composición corporal (el componente metabólicamente activo, la masa libre de grasa), estas diferencias entre individuos obesos y no obesos desaparecen, lo que sugiere que el gasto energético en individuos obesos no se altera. Esto puede explicarse porque la masa libre de grasa (principal determinante del gasto energético basal) aumenta en forma paralela con el aumento de la masa grasa. Sin embargo, una pregunta importante, aún sin respuesta, es si el GEA es más bajo en individuos obesos como consecuencia de la disminución en la actividad física en general o debido a un menor gasto de energía durante la actividad física. Por lo tanto, según el conocimiento actual, el GEA y la TIA se reducen en las personas obesas, principalmente debido a un estilo de vida poco saludable (poca actividad física y mayor consumo de grasa) ${ }^{(14)}$.

Un aspecto muy importante y objeto de investigación es la posibilidad de que el gasto energético esté alterado en ciertos tipos de obesidad. Los fenotipos de obesidad recientemente descritos como la obesidad normo - peso (normal - weight obese, NWO, por su sigla en inglés $)^{(15)}$ o la obesidad sarcopénica ${ }^{(16)}$ podrían presentar un gasto energético alterado en comparación con aquellos que no son obesos o son obesos con una composición corporal normal. El obeso normo-peso es aquella persona que tiene un exceso de tejido adiposo (masa grasa $>30 \%$ según DXA) con un Índice de Masa Corporal (IMC) normal. En estas personas la diferencia en el gasto energético podría explicarse por la menor masa libre de grasa. Sin embargo, no hay estudios que confirmen esta hipótesis.

La obesidad sarcopénica se define como una relación Índice de masa grasa / Índice de masa libre de grasa (fat mass index / the fat-free mass index, FMI / FFMI) $>$ percentil 95 de las referencias según la población y específicas para sexo, IMC y etnia (esta definición ha evolucionado desde la de Baumgartner et al. ${ }^{(17)}$. En teoría, el aumento del tejido libre de grasa sería insuficiente para soportar el exceso de masa grasa en el individuo obeso sarcopénico. Si la masa libre de grasa es un determinante importante del gasto energético en reposo y disminuye en la obesidad sarcopénica, es razonable esperar que el gasto energético sea más bajo en estos individuos, lo que implica que el sedentarismo puede agravar el efecto de la disminución del gasto energético y promover el ciclo vicioso hipotético de la obesidad (mayor peso corporal / menor actividad física / menor FFM / aumento de peso corporal $^{(18)}$ (Figura 2). Además, la obesidad sarcopénica se asocia a un aumento de las comorbilidades relacionadas con el exceso de adiposidad, como la resistencia a la insulina, la hipertensión y la dislipidemia ${ }^{(19)}$; la sobrestimación de los requisitos de energía en esta población podría llevar a un mayor aumento de su masa grasa y, por lo tanto, afectar negativamente esta condición ${ }^{(20)}$. Se sugiere que los estudios futuros comparen los componentes del gasto energético con posibles anormalidades fisiológicas asociadas a cada fenotipo ${ }^{(14)}$.

En conclusión, se sabe poco sobre el GEA en la población obesa y en especial en ciertos fenotipos de obesidad por lo que se necesitan estudios adicionales que comparen la GEA entre individuos obesos y no obesos. La evidencia actual no respalda la hipótesis de que la obesidad es consecuencia de una alteración en 


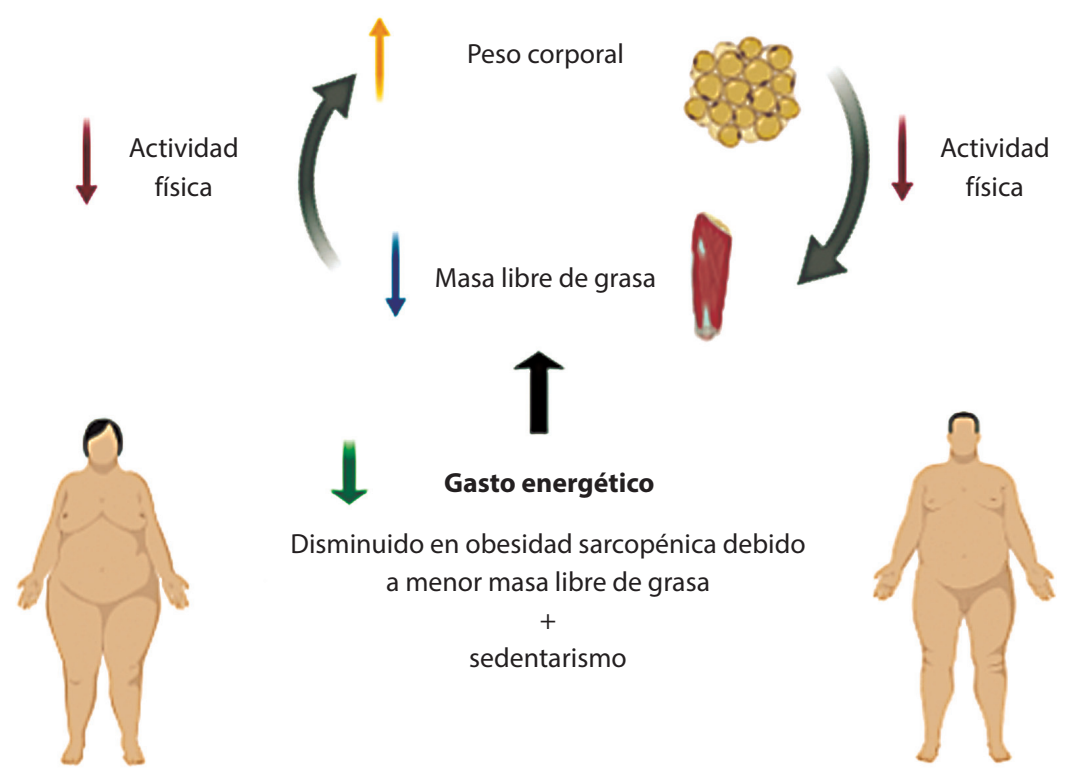

Figura 2. El sedentarismo puede agravar el efecto de la disminución del gasto energético (GE) y promover el ciclo vicioso hipotético de la obesidad (mayor peso corporal / menor actividad física / menor masa libre de grasa / aumento de peso corporal).

el gasto energético. Estos hallazgos son especialmente relevantes para los nutricionistas y otros profesionales de la salud que atienden a personas obesas que creen que su exceso de peso corporal está relacionado con un «metabolismo lento» o un gasto energético alterado.

\section{EL PAPEL DEL EJERCICIO EN LA PÉRDIDA DE PESO}

Para lograr la pérdida de peso la Organización Mundial de la Salud (OMS) ${ }^{(21)}$ y sociedades científicas internacionales como la Academia Nacional Norteamericana de Nutrición y Dietética ${ }^{(22)}$, la Asociación Americana de Diabetes $(\mathrm{ADA})^{(23)}$ recomiendan el ejercicio como parte integral de cualquier programa de pérdida de peso. El estado actual de la evidencia respalda la importancia del ejercicio (actividad física estructurada y planificada) en la mejora de la salud cardiovascular, la sensibilidad a la insulina, el control glucémico de la diabetes tipo 2, la presión arterial y la depresión ${ }^{(22)}$. Sin embargo, cabe preguntar si el ejercicio en sí mismo contribuye a la pérdida de peso y los esfuerzos de mantenimiento del peso corporal ${ }^{(24)}$.

\section{¿EL EJERCICIO MEJORA LOS ESFUERZOS EN LA PÉRDIDA DE PESO?}

Con el objetivo de determinar las estrategias para perder peso, se han realizado investigaciones que evalúan el impacto de solo ejercicio, el ejercicio más la restricción dietética o la restricción dietética sola ${ }^{(24)}$. Según una revisión sistemática de estudios con un seguimiento mínimo de 1 año, los sujetos que usaron solo el ejercicio para la reducción de peso experimentaron una pérdida de peso mínima ${ }^{(25)}$.

El estudio de Redman et al., tuvo como objetivo determinar si el ejercicio mejora los esfuerzos de la pérdida de peso. Treinta y seis pacientes con sobrepeso se dividieron en dos grupos, un grupo realizó ejercicio más restricción calórica y el otro solo restricción calórica. Se mostró que el déficit calórico se mantuvo constante durante los 6 meses del estudio en ambos grupos. Se logró una pérdida de peso de $10 \%$ en este periodo en ambos grupos, sin una diferencia estadísticamente significativa en el porcentaje de pérdida de grasa corporal. Sin embargo, el grupo de ejercicio tuvo el beneficio adicional de mejorar la aptitud aeróbica ${ }^{(26)}$.

En otro estudio de Ross et al. se estudiaron 52 hombres obesos (IMC $31,3 \pm 2,0 \mathrm{~kg} / \mathrm{m}^{2}$ ) y se evidenció una disminución del peso corporal de 7,5 kg durante 3 meses en el grupo de solo ejercicio ( 16 hombres) el cual fue comparable a la del grupo con restricción calórica. La duración del ejercicio se basó en el objetivo de un gasto diario de energía de 700 calorías ( $60 \mathrm{~min}$ / día). Los resultados de este estudio sugieren que puede ser necesario realizar un ejercicio superior a las recomendaciones internacionales mínimas de salud de 150 min / semana para lograr una pérdida de peso clínica- 
mente significativa. Estos resultados fueron confirmados por otros estudios como el Midwest Exercise Trial 2 realizado en 141 hombres y mujeres con sobrepeso ${ }^{(27)}$.

Con el objetivo de evaluar la hipótesis de que el ejercicio atenúa las reducciones en la masa magra, la fuerza muscular, la densidad mineral ósea y el $\dot{\mathrm{V}}{ }_{2 \text { máx }}$ que acompañan la pérdida de peso moderada inducida por la restricción calórica, Weiss et al. estudiaron 52 hombres y mujeres obesos y sedentarios. El estudio mostró no solo una pérdida de peso efectiva ( $7 \%$ durante 16,8 semanas) con ejercicio exclusivamente, sino también la preservación de la masa corporal magra y la mejora del consumo máximo de oxígeno $\left(\dot{\mathrm{V}}_{2 \operatorname{maxx}}\right)$ en comparación con solo restricción calórica. Además, al igual que los estudios anteriores que han demostrado la pérdida de peso con el ejercicio, la cantidad de ejercicio fue importante: 7,4 $\pm 0,5$ horas / semana ${ }^{(28)}$.

En conclusión, el ejercicio juega un papel limitado en la pérdida de peso. Se necesitan intervenciones de ejercicio mayores a las recomendaciones generales (150 min / semana de caminata rápida). El fracaso en la pérdida de peso puede deberse a que las personas obesas con frecuencia tienen dificultades para realizar el ejercicio necesario para crear un déficit energético suficiente, el cual se puede contrarrestar fácilmente al aumentar la ingesta energética ${ }^{(24)}$. Según el Colegio Americano de Medicina Deportiva y la Asociación Americana de Diabetes «los niveles recomendados de actividad física pueden ayudar a producir pérdida de peso. Sin embargo, se pueden requerir hasta $60 \mathrm{~min} /$ día cuando se depende solo del ejercicio para bajar de peso ${ }^{(29)}$.

\section{ACTIVIDAD FÍSICA Y MANTENIMIENTO DEL PESO CORPORAL}

La actividad física puede ser un componente importante en el mantenimiento del peso después de la pérdida de peso ${ }^{(24)}$. Schoeller et al. realizaron un estudio prospectivo con el objetivo de saber si la actividad física después de la pérdida de peso es capaz de predecir el mantenimiento del peso corporal. Para ello, se midió el gasto total energético con el método de agua doblemente etiquetada en 32 mujeres obesas. Se mostró que la actividad física en el rango de 11- $12 \mathrm{kcal} / \mathrm{kg} /$ día (900 calorías / día para una mujer de $81 \mathrm{~kg}$ ) puede ser importante para prevenir el aumento de peso ${ }^{(30)}$.

Una revisión sistemática de la literatura realizada por Fogelholm y Kukkonen-Harjula sugiere que un aumento en el gasto energético de $1.500-2.000$ calorías / semana está asociado al mantenimiento del peso $^{(31)}$. Además, un estudio prospectivo con un seguimiento de 33 años, mostró que los hombres que mantuvieron una actividad > $150 \mathrm{~min} /$ semana ganaron $5,6 \mathrm{~kg}$ en comparación con 9,1 kg en hombres menos activos, con una tendencia aún más significativa ( $3,8 v \mathrm{~s}$ $9,5 \mathrm{~kg}$ ) entre las mujeres ${ }^{(32)}$.

En conclusión, el ejercicio constante de una duración mayor a las recomendaciones básicas para la salud (150 min / semana de ejercicio de intensidad moderada) contribuye a la pérdida de peso y al mantenimiento de peso a largo plazo. La actividad física de todo tipo, incluidos los ejercicios aeróbicos, los de resistencia, de flexibilidad y la reducción del tiempo sedentario, claramente resultan en múltiples beneficios para la salud de las personas obesas y deben incluirse en cualquier recomendación de estilo de vida ${ }^{(22,29)}$.

Recomendar a las personas hacer ejercicio durante períodos de tiempo más largos cada día puede ayudar a mejorar la pérdida y el mantenimiento del peso. Se debe tener en cuenta que existen diferencias individuales que pueden depender de factores genéticos, el IMC, la composición corporal, la intensidad, la duración del ejercicio y el tipo de ejercicio. Es un desafío para algunos pacientes lograr hacer, incluso, ejercicios pequeños de manera consistente todos los días; en consecuencia, es importante no centrarse en la posibilidad de perder peso como el único resultado del ejercicio, sino explicar que el ejercicio puede contribuir a los esfuerzos de pérdida de peso y obtener una gran cantidad de otros beneficios relacionados con la salud. Este enfoque reducirá la probabilidad de que los pacientes utilicen la falta de pérdida de peso como una razón para descontinuar su programa de ejercicio.

\section{EFECTO DE LA ACTIVIDAD FÍSICA Y EL EJERCICIO EN LAS ENFERMEDADES CRÓNICAS}

Los beneficios del ejercicio sobre enfermedades crónicas como la diabetes de tipo 2, las enfermedades cardiovasculares, la osteoporosis y el cáncer, han sido tema de estudio. Estudios observacionales muestran una asociación entre los niveles más altos de actividad física (toda actividad que genere gasto energético) y tasas más bajas de enfermedades crónicas. Por el contrario, la inactividad física se asocia a una esperanza de vida reducida. Un estudio publicado en The Lancet en 2012, y realizado en 2008, estima que la proporción de adultos inactivos en el mundo es de $31 \%$ y que la inactividad física es responsable de $9 \%$ de la mortalidad prematura en 
todo el mundo, en particular por enfermedad coronaria y diabetes, enfermedades secundarias a la obesidad ${ }^{(33)}$.

La actividad física tiene un efecto positivo en el control de la diabetes tipo 2, en la prevención primaria en sujetos en riesgo (personas con sobrepeso, obesidad en particular abdominal o intolerancia a la glucosa) y en la prevención terciaria de complicaciones. La evidencia muestra que también reduce los niveles de triglicéridos, aumenta los niveles de colesterol HDL, disminuye el riesgo de desarrollar obesidad y ayuda a perder peso.

La actividad física favorece una mejor captación de glucosa por parte del músculo esquelético a través de los transportadores de glucosa GLUT 4, un mejor uso de los ácidos grasos libres por el músculo esquelético y una mayor sensibilidad a la insulina. De este modo, permite reducir la grasa y la masa visceral, sin que necesariamente implique una pérdida de peso. La actividad física ayuda también a reducir el estado inflamatorio frecuente en la obesidad al reducir los marcadores de inflamación. El ejercicio físico permite una reducción de la glucemia mediante un efecto inmediato a través del reclutamiento de GLUT 4 y un efecto prolongado al activar la AMPquinasa, enzima que aumenta el gasto calórico y reduce la acumulación de lípidos intramusculares ${ }^{(34)}$.

El Nurse Health Study (NHS) es un estudio observacional iniciado en los Estados Unidos de América en 1976, el cual realizó un seguimiento de más de 115,000 mujeres entre 30 y 55 años de edad. El riesgo relativo de muerte en comparación con las mujeres delgadas y activas (más de $3 \mathrm{~h}, 30$ min de actividad física por semana) fue de 1,55 para las mujeres delgadas e inactivas, 1,91 para las mujeres obesas activas y 2,42 para las mujeres obesas inactivas. Un sub-análisis del NHS siguió 50.277 mujeres de 1992 a 1998 y encontró que cada hora de caminata enérgica disminuía el riesgo de obesidad en $24 \%$ y el riesgo de desarrollar diabetes tipo 2 en $34 \%{ }^{(35)}$.

La actividad física es también un medio eficaz en la prevención primaria y terciaria de enfermedades cardiovasculares (insuficiencia cardíaca, enfermedad coronaria, etc.), así como en su tratamiento y en la reducción de la presión arterial. Cuanto mayor sea el nivel de actividad física, menor será el riesgo. El estudio americano observacional Women's Health Initiative Observational Study estudió a 73.743 mujeres entre 50 y 79 años, sin enfermedad cardiovascular, por un promedio de 3,2 años. Se estudió la relación entre la ocurrencia de eventos cardiovasculares (infarto de miocardio, muerte por enfermedad coronaria, accidente cerebrovascular, primer evento cardiovascular) y actividad física. El riesgo relativo de enfermedad cardiovascular disminuyó con el aumento de la actividad física de manera similar al caminar o con la actividad física extenuante ${ }^{(36)}$.

La actividad física tiene un papel preventivo en la aparición de osteporosis y en el riesgo de fractura osteoporótica en mujeres y en hombres. La actividad física regular hace posible que las mujeres con riesgo de osteoporosis reduzcan el riesgo de una fractura osteoporótica. Esta disminución puede explicarse por mejores capacidades musculares y cardiorrespiratorias, mejor equilibrio, mejor control de la caída y mayor resistencia ósea en mujeres que practican actividad física en comparación con mujeres sedentarias. Un estudio de Recker et al. siguió a 156 estudiantes sanos durante 5 años y mostró que la actividad física y la ingesta suficiente de calcio permitieron un aumento en la ganancia de densidad ósea. La actividad física combinada con una ingesta suficiente de calcio, puede por lo tanto prevenir el riesgo de osteoporosis ${ }^{(37)}$. Las actividades de resistencia aeróbica (caminar, ejercicios elípticos, aeróbicos acuáticos), actividades dinámicas y de fortalecimiento muscular para mantener o aumentar la densidad mineral ósea y actividades para mejorar el equilibrio y la fuerza muscular para limitar las caídas, son las actividades físicas recomendadas para el tratamiento de la osteoporosis ${ }^{(38)}$.

Con respecto al cáncer, la actividad física se asocia a la disminución de ciertos tipos de cáncer como el de colon, pulmón, próstata, endometrio y de mama. Existe una relación dosis - respuesta, con el aumento en el nivel de actividad física que permite una mayor disminución en el riesgo en casi todos los tipos de cáncer. Un reciente meta-análisis de 52 estudios mostró que las personas que son físicamente activas tienen un riesgo $20 \%$ a $30 \%$ menor de tener cáncer de colon en comparación con las personas menos activas ${ }^{(39)}$. En la cohorte del NHS, la actividad física de intensidad moderada produjo una disminución de $33 \%$ en el riesgo de cáncer de colon, mientras que la actividad física de alta intensidad produce una disminución del riesgo de $46 \%$ de cáncer de colon. Con respecto al cáncer de mama, diversos estudios han demostrado una disminución en el riesgo de desarrollar este tipo de cáncer con el aumento de la actividad física, incluido un meta-análisis en 2006 que mostró una reducción del riesgo de $30 \%$ a $40 \%$. La mayoría de estos estudios informan una relación dosis-respuesta.

Diferentes estudios han investigado los mecanismos que pueden explicar la disminución del riesgo de desarrollar cáncer a través de la actividad física. La actividad física puede disminuir los niveles circulan- 
tes de insulina, IGF-1 y hormonas sexuales, cuya alta concentración promueve el desarrollo de ciertos tipos de cáncer de mama, próstata y colon. Además, la actividad física reduce la grasa corporal y el sobrepeso es un factor de riesgo para desarrollar ciertos tipos de cáncer. La actividad física promueve la motilidad intestinal, lo que puede explicar en parte el efecto protector sobre el cáncer de colon.

La actividad física también tiene un impacto en la ansiedad y la depresión. Un meta-análisis muestra que la actividad física permite una reducción significativa de los rasgos de ansiedad, especialmente en personas con altos niveles de ansiedad, mala condición física, pero también en personas no ansiosas ${ }^{(40)}$.

\section{MECANISMOS QUE EXPLICAN LOS EFECTOS BENÉFICOS DEL EJERCICIO EN LA SALUD: EL PAPEL DE LAS MIOCINAS Y EL MÚSCULO COMO ÓRGANO ENDOCRINO}

El ejercicio estimula la liberación de proteínas con funciones autocrinas, paracrinas y endocrinas producidas en el músculo esquelético denominadas miocinas ${ }^{(41)}$. Su principal función fisiológica es proteger la funcionalidad y mejorar la capacidad de ejercicio del músculo esquelético. Las miocinas controlan los procesos adaptativos en el músculo esquelético cuando actúan como reguladores autocrinos y paracrinos de la oxidación energética, la hipertrofia, la angiogénesis, los procesos inflamatorios y la regulación de la matriz extracelular. Las funciones endocrinas atribuidas a las miocinas están involucradas en la regulación del peso corporal, la inflamación de bajo grado, la sensibilidad a la insulina, supresión del crecimiento tumoral y la mejora de la función cognitiva ${ }^{(42,43,44)}$.

Durante el ejercicio el músculo esquelético tiene la capacidad de expresar y reprimir genes a través de señales intracelulares y extracelulares. Las principales señales intracelulares son segundos mensajeros como el aumento del calcio intracelular, la depleción de la adenosina trifosfato (ATP), la nicotinamida adenina dinucleótido (NAD) y el aumento de especies reactivas de oxígeno (ROS) (Figura 3). También lo realiza a través de señales extracelulares como la presión de oxígeno extracelular, señales endocrinas y estímulos mecánicos, entre otros ${ }^{(41)}$. El principal regulador de la actividad génica, durante el ejercicio, es el PCG-1 $\alpha$ (coactivador $1 \alpha$ del receptor activador de la proliferación de peroxisomas gamma). Se trata de una proteína necesaria para la transcripción de genes que induce, entre otras funciones, los procesos adaptativos del músculo esquelético ${ }^{(41)}$. El ejercicio aumenta la activación del gen del PGC-1 $\alpha$ en el músculo esquelético $^{(45)}$. Algunas respuestas asociadas al PCG-1 $1 \alpha$ y, que podrían explicar los efectos benéficos del ejercicio en la salud, son el aumento de la expresión de receptores de insulina, la captación de ácidos grasos y glucosa, el almacenamiento de glucógeno y la biogénesis mitocondrial. El PCG-1 $\alpha$ favorece también la síntesis de miocinas con efecto endocrino sobre el mismo músculo esquelético y distintos órganos. La Figura 3 muestra las principales miocinas secretadas por el músculo esquelético y su relación con otros órganos como el tejido adiposo, el hígado, el páncreas, el intestino y el cerebro. Algunas miocinas como el LIF (factor inhibidor de la leucemia) y la IL-6 (interleucina 6) participan en la hipertrofia muscular y la miogénesis interactuando a través de las células satélites musculares (efecto paracrino). Miocinas como IL-6, LIF, FGF21 (Factor de crecimiento fibroblástico 21) y el BDNF (factor neurotrófico derivado del cerebro) y favorecen una mayor captación de ácidos grasos y la hidrólisis de triglicéridos en el tejido adiposo. El objetivo es garantizar un aporte continuo y suficiente de sustratos para poder proporcionar las reservas energéticas y asegurar la reparación tisular. Miocinas como IL-6, LIF y otras (IL-15, FGF- 21, FNDC5, SPARC) favorecen la expresión muscular de la GLUT4 (proteína transportadora de glucosa mediada por insulina) por mecanismos independientes de la insulina, lo que reduce las concentraciones plasmáticas de glucosa durante el ejercicio y hasta $24 \mathrm{~h}$ posterior a este ${ }^{(41)}$. Estos efectos refuerzan el impacto, ya bien conocido, que tiene el ejercicio físico sobre la prevención y el manejo de la diabetes mellitus.

La IL-6 también tiene efectos endocrinos en el hígado, el tejido adiposo y el sistema inmunitario, y media la comunicación entre las células L intestinales y los islotes pancreáticos. Otras miocinas como los factores osteogénicos IGF-1 y FGF-2; FSTL-1, mejoran la función endotelial del sistema vascular; y la miocina irisina dependiente de PGC-1 $\alpha$, que impulsa el desarrollo similar a la grasa parda ${ }^{(41)}$.

La producción de la mayoría de las citoquinas en el músculo esquelético depende de la contracción. Por lo tanto, la inactividad física probablemente conduce a una respuesta alterada de estas proteínas lo que podría explicar la asociación entre el sedentarismo y las enfermedades crónicas ${ }^{(4)}$. Este es un campo muy amplio y de gran auge en investigación. Se busca que algunos metabolitos reguladores derivados del músculo, o la 


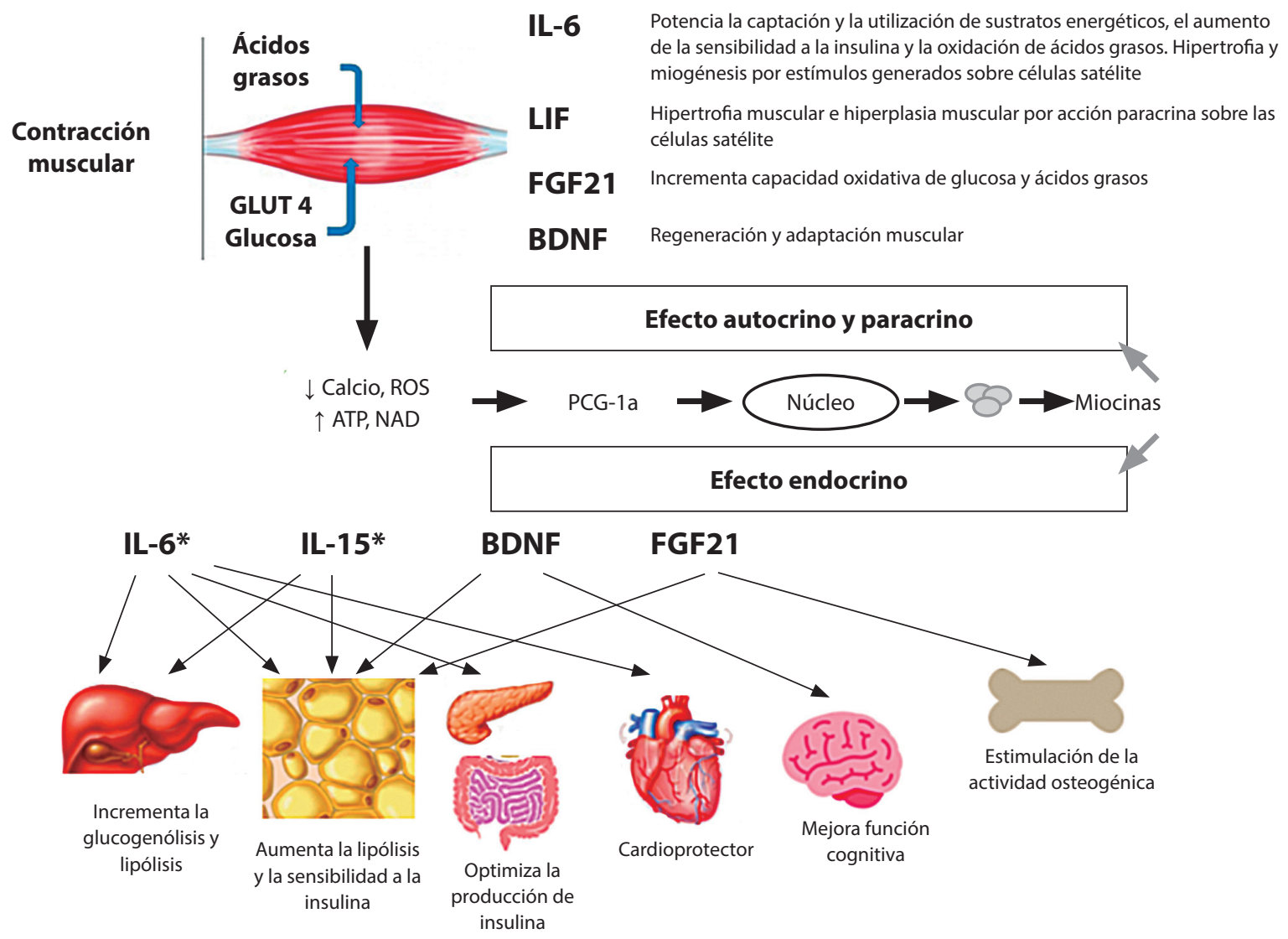

Figura 3. El papel de las miocinas en los efectos benéficos para la salud. LIF (factor inhibidor de la leucemia), IL-6 (interleucina 6), FGF21 (Factor de crecimiento fibroblástico 21), BDNF (factor neurotrófico derivado del cerebro), GLUT4 (proteína transportadora de glucosa mediada por insulina), PCG-1a (coactivador 1a del receptor activador de la proliferación de peroxisomas gamma), adenosina trifosfato (ATP), la nicotinamida adenina dinucleótido (NAD) y el aumento de especies reactivas de oxígeno (ROS). Adaptado de León HH et al. ${ }^{(43)}$.

producción de miocinas modificadas, puedan ser estrategias prometedoras a futuro para el tratamiento de enfermedades crónicas.

\section{ACTIVIDAD FÍSICA Y OBESIDAD EN NIÑOS Y ADOLESCENTES}

El sedentarismo ha sido relacionado como una de las causas de la obesidad infantil ${ }^{(46)}$. La actividad física es uno de los pilares tanto para la prevención como para el tratamiento de la obesidad en niños y adolescentes.

Diversas sociedades científicas han establecido múltiples recomendaciones de actividad física para niños y adolescentes. Las más recientes guías de la OMS sobre actividad fisica, sedentarismo y sueño en niños menores de 5 años ${ }^{(47)}$, señalan que los lactantes menores de 12 meses deben permanecer al menos 30 minutos en posición prono, entre los 12 y 24 meses se deben alcanzar al menos 180 minutos de actividades fisicas de cualquier intensidad; y a partir de los 3 años se debe promover, a través de actividades que inciten el juego dinámico, que los niños sean físicamente activos, por lo menos 3 horas diarias, de las cuales mínimo 1 hora debe ser de intensidad moderada a vigorosa, esto permitirá favorecer el crecimiento y desarrollo infantil ${ }^{(48)}$.

Las recomendaciones de un gran número de sociedades científicas adhieren las de la OMS $(2010)^{(49)}$ e incluyen un mínimo de 60 minutos diarios de actividad física aeróbica en su mayoría de intensidad moderada o vigorosa para niños y adolescentes entre 5 y 17 años de edad. Además, se recomienda que al menos 3 veces por semana se incluyan actividades de intensidad vigorosa para fortalecer los huesos y músculos, y favorecer el crecimiento.

Los adolescentes con peso normal y actividad física regular, tienen menor porcentaje de tejido adiposo y mayor proporción de masa muscular que quienes no la realizan de la misma forma ${ }^{(49)}$. En consecuencia, dado 
que los niños y adolescentes con obesidad tienen una baja tolerancia al ejercicio, debido a la gran demanda de oxígeno por el exceso de peso ${ }^{(50)}$, se debe iniciar actividad fisica de manera paulatina, empezando con ejercicios ligeros y cortos durante 30 minutos hasta alcanzar los 60 minutos diarios, mínimo 3 y hasta alcanzar 7 días a la semana como lo recomienda la OMS. Incluso en niños y adolescentes con alto riesgo de enfermedad cardiovascular se recomienda incluir el ejercicio como parte del tratamiento ${ }^{(51)}$.

\section{CONCLUSIÓN}

En conclusión, el ejercicio y la actividad física son importantes para mantener la pérdida de peso, principalmente a largo plazo y son la clave para preservar la masa corporal magra durante una alimentación con restricción energética. El papel del ejercicio trasciende el control del peso, conlleva efectos benéficos demostrados para la salud, en especial para el control de las enfermedades crónicas. Aunque el músculo esquelético fue considerado durante mucho tiempo un órgano encargado solo de la locomoción, el almacenamiento de proteínas y la generación de calor, hoy se sabe que actúa como une órgano endocrino a través de las miocinas. Estas proteínas pueden mediar los efectos protectores del ejercicio muscular, con respecto a enfermedades asociadas a un estilo de vida físicamente inactivo. La práctica regular de actividades físicas y deportivas relacionadas con una alimentación adecuada es, por lo tanto, uno de los elementos esenciales del tratamiento de la obesidad.

\section{Financiación}

El presente artículo no tuvo financiación.

\section{Conflicto de intereses}

Los autores declaran no tener conflicto de intereses.

\section{Declaración de autoría}

DC, ALMP, LL participaron en la concepción, la redacción y revisión del artículo. Los autores revisaron el artículo y validaron su versión final.

\section{Referencias bibliográficas}

1. Departamento de la Prosperidad Social. Instituto Colombiano de Bienestar Familiar. ENSIN: Encuesta Nacional de la Situación Nutricional [Internet]. Colombia; 2015 [Fecha de consulta:20 Julio de 2019]. Disponible en https://www.icbf. gov.co/bienestar/nutricion/encuesta-nacional-situacionnutricional.

2. Organización de la Naciones Unidas. Más hambrientos y más obesos en América Latina en medio de la desigualdad [Internet]. Noviembre 7 de 2018. [Consultado: 20 de julio de 2019]. Disponible en https://news.un.org/es/ story/2018/11/1445101

3. Global Burden of Metabolic Risk Factors for Chronic Diseases Collaboration (BMI Mediated Effects), Lu Y, Hajifathalian K, Ezzati M, Woodward M, Rimm EB, et al. Metabolic mediators of the effects of body-mass index, overweight, and obesity on coronary heart disease and stroke: a pooled analysis of 97 prospective cohorts with 1.8 million participants. Lancet. 2014; 383(9921):970-83.

4. McGee DL. Body mass index and mortality: a meta-analysis based on person-level data from twenty-six observational studies. Ann Epidemiol. 2005;15(2):87-97.

5. Basdevant A, Aron-Wisnewski J, Clément K. Définitions des obésités. Traité Médecine et Chirurgie de l'obésite. Médecine Sciences Publications. Lavoisier; 2011. p. 3-8.

6. LaMonte MJ, Blair SN. Physical activity, cardiorespiratory fitness, and adiposity: contributions to disease risk. Curr Opin Clin Nutr Metab Care. 2006; 9(5): 540-6.

7. Wiklund $P$. The role of physical activity and exercise in obesity and weight management: Time for critical appraisal. J Sport Health Sci. 2016; 5(2): 151-4.

8. Ng M, Fleming T, Robinson M, Thomson B, Graetz N, Margono C, et al. Global, regional, and national prevalence of overweight and obesity in children and adults during 19802013: a systematic analysis for the Global Burden of Disease Study 2013. Lancet. 2014; 384(9945): 766-81.

9. Blair SN, Archer E, Hand GA. Commentary: Luke and Cooper are wrong: physical activity has a crucial role in weight management and determinants of obesity. Int J Epidemiol. 2013; 42(6):1836-8.

10. Hill JO, Peters JC. Commentary: physical activity and weight control. Int J Epidemiol. 2013; 42(6):1840-2.

11. Luke A, Cooper RS. Physical activity does not influence obesity risk: time to clarify the public health message. Int J Epidemiol. 2013; 42(6):1831-6.

12. Westerterp KR. Assessment of physical activity: a critical appraisal. Eur J Appl Physiol. 2009; 105(6): 823-8.

13. Jensen MD, Ryan DH, Apovian CM, Ard JD, Comuzzie AG, Donato KA, et al. 2013 AHA/ACC/TOS guideline for the management of overweight and obesity in adults: a report of the American College of Cardiology/American Heart Association Task Force on Practice Guidelines and The Obesity Society. Circulation. 2014; 129(25 Suppl 2): S102-38.

14. Carneiro IP, Elliott SA, Siervo M, Padwal R, Bertoli S, Battezzati A, et al. Is Obesity Associated with Altered Energy Expenditure? Adv Nutr. 2016;7(3): 476-87. 
15. Di Renzo L, Del Gobbo V, Bigioni M, Premrov MG, Cianci R, De Lorenzo A. Body composition analyses in normal weight obese women. Eur Rev Med Pharmacol Sci. 2006;10(4):191-6.

16. Prado CM, Siervo M, Mire E, Heymsfield SB, Stephan BC, Broyles S, et al. A population-based approach to define bodycomposition phenotypes. Am J Clin Nutr. 2014;99(6):1369-77.

17. Baumgartner RN. Body composition in healthy aging. Ann N Y Acad Sci. 2000; 904: 437-48.

18. Ward J. Sarcopenia and sarcopenic obesity: is it time the health system accepted fitness of older people as a health responsibility? Australas J Ageing. 2011;30(2): 61-2.

19. Prado CM, Wells JC, Smith SR, Stephan BC, Siervo M. Sarcopenic obesity: a critical appraisal of the current evidence. Clin Nutr. 2012; 31(5):583-601.

20. Prado CM, Heymsfield SB. Lean tissue imaging: a new era for nutritional assessment and intervention. JPEN J Parenter Enteral Nutr. 2014; 38(8): 940-53.

21. Organisation Mondiale de la Santé. Stratégie sur l'activité physique pour la Région européenne de l'OMS 20162025 [Internet]. Comité Régional de L'europe SoixanteCinquiéme Session ;14-17 septembre 2015. [Consultado el : 1 de Agosto de 2019] Disponible en http://www.euro. who.int/_data/assets/pdf_file/0010/283807/65wd09f_ PhysicalActivityStrategy_150474_withCover.pdf.

22. Colberg SR, Sigal RJ, Yardley JE, Riddell MC, Dunstan DW, Dempsey PC, et al. Physical activity/exercise and diabetes: a position statement of the American Diabetes Association. Diabetes Care. 2016;39(11):2065-79.

23. Handelsman Y, Bloomgarden ZT, Grunberger G, Umpierrez G, Zimmerman RS, Bailey TS, et al. American Association of Clinical Endocrinologists and American College of Endocrinology- clinical practice guidelines for developing a diabetes mellitus comprehensive care plan-2015. Endocr Pract. 2015; 21(Suppl 1):1-87.

24. Cox CE. Role of Physical Activity for Weight Loss and Weight Maintenance. Diabetes Spectr. 2017; 30(3):157-60.

25. Franz MJ, VanWormer JJ, Crain AL, Boucher JL, Histon T, Caplan W, et al. Weight-loss outcomes: a systematic review and Meta-analysis of weight-loss clinical trials with a minimum 1-year follow-up. J Am Diet Assoc. 2007;107(10):1755-67.

26. Redman LM, Heilbronn LK, Martin CK, Alfonso A, Smith SR, Ravussin E, et al. Effects of calorie restriction with or without exercise on body composition and fat distribution. J Clin Endorcrinol Metab. 2007;92(3):865-72.

27. Weiss EP, Jordan RC, Frese EM, Albert Sg, Villareal DT. Effects of weight loss on lean mass, strength, bone and aerobic capacity. Med Sci Sports Exerc. 2017;49(1):206-17.

28. Donnelly JE, Honas JJ, Smith BK, Mayo MS, Gibson CA, Sullivan DK, et al. Aerobic exercise alone results in clinically significant weight loss for men and women: Midwest Exercise Trial 2. Obesity ( Silver Spring). 2013;21(3):E219-E28.

29. Colberg SR, Sigal RJ, Fernhall B, Regensteiner JG, Blissmer BJ, Rubin RR, et al. Exercise and type 2 diabetes: the
American College of Sports Medicine and the American Diabetes Association: joint position statement. Diabetes Care. 2010;33(12): e147-67.

30. Schoeller DA, Shay K, Kushner RF. How much physical activity is needed to minimize weight gain in previously obese women? Am J Clin Nutr. 1997; 66(3): 551-6

31. Fogelholm M, Kukkonen-Harjula K. Does physical activity prevent weight gain: a systematic review. Obes Rev. 2000; 1(2): 95-111.

32. Moholdt T, Wisløff U, Lydersen S, Nauman J. Current physical activity guidelines for health are insufficient to mitigate long-term weight gain: more data in the fitness versus fatness debate (The HUNT study, Norway). Br J Sports Med. 2014;48(20):1489-96.

33. Lee IM, Shiroma EJ, Lobelo F, Puska P, Blair SN, Katzmarzyk $\mathrm{PT}$, et al. Effect of physical inactivity on major non-communicable diseases worldwide: an analysis of burden of disease and life expectancy. Lancet. 2012;380(9838):219-29.

34. Raguso CA, Spada A, Jornayvaz FR, Philippe J. Lactivité physique dans la prévention et le contrôle du diabète. Rev Med Suisse. 2007;3(114).

35. Gallois P, Vallée JP, Le Noc Y. L'activité physique: pourquoi? pour qui? comment la prescrire? Médecine. 2006;2(1):20-4.

36. Manson JE, Greenland P, LaCroix AZ, Stefanick ML, Mouton $\mathrm{CP}$, Oberman A, et al. Walking Compared with Vigorous Exercise for the Prevention of Cardiovascular Events in Women. N Engl J Med. 2002;347(10):716-25.

37. Recker RR, Davies KM, Hinders SM, Heaney RP, Stegman MR, Kimmel DB. Bone gain in young adult women. JAMA. 1992;268(17):2403-8.

38. Depiesse F, Cayrac C. Ostéoporose et activité physique. En: F. Depiesse, O. Coste. Prescription des activités physiques: en prévention et en thérapeutique. $2^{\text {nd }}$ Edition. Elsevier Masson; 2016. p.159-94.

39. Chan AT, Giovannucci EL. Primary Prevention of Colorectal Cancer. Gastroenterology. 2010;138(6):2029-2043.e10.

40. Conn VS. Anxiety outcomes after physical activity interventions: meta-analysis findings. Nurs Res. 2010;59(3):224-31.

41. Pedersen BK, Steensberg A, Fischer C, Keller C, Keller P, Plomgaard P, et al. Searching for the exercise factor: Is IL-6 a candidate? J Muscle Res Cell Motil. 2003; 24(2-3):113-9.

42. León-Ariza HH, Mendoza-Navarrete MP, Maldonado-Arango MI, Botero-Rosas DA. Miocinas y regulación metabólica, una revisión sistemática. Apunts Med L'Esport. 2018; 53(200):155-62.

43. León HH, Melo CE, Ramírez JF. Role of the myokines production through the exercise. J Sport Heal Res. 2012;4(2):157-66.

44. Pedersen BK, Febbraio MA. Muscles, exercise and obesity: skeletal muscle as secretory organ. Nat Rev Endocrinol. 2012;8(8):457-65.

45. Norrbom J, Sundberg CJ, Ameln H, Kraus WE, Jansson E, Gustafsson T . PGC-1alpha mRNA expression is influenced 
by metabolic perturbation in exercising human skeletal muscle. J Appl Physiol (1985). 2004;96(1):189-94.

46. da Silva AP, Maia TC, Silva DC, et al. Prevalence of overwight and obesity and associated factors in school children and adolescents in a medium sized Brazilian city. Clinics 2018;73:e438.

47. World Health Organization (WHO). Guidelines on Physical Activity, Sedentary Behaviour and Sleep for Children under 5 years of age. WHO 2019.

48. Physical Activity Guidelines for Americans, [Internet]. 2nd edition 2018 [Consultado: 1 de Agosto de 2019]. Disponible en. https://health.gov/paguidelines/second-edition/pdf/ Physical_Activity_Guidelines_2nd_edition.pdf

49. Organización Mundial de la Salud (OMS). Recomendaciones mundiales de actividad sobre la actividad física en la salud. [Internet]. OMS 2010 [Consultado: 1 de Agosto de 2019]. Disponible en: https://www.who.int/dietphysicalactivity/ factsheet_recommendations/es/

50. Kleinman R, Greer F, editors. Pediatric Obesity. In: Pediatric Nutrition, AAP $7^{\text {th }}$ edition 2014.

51. Corkins MR, Balint J, Bobo E, Plogsted S, Yaworski JA, editors. Obesity and Metabolic Syndrome. In: The ASPEN Pediatric Nutrition Support Core Curriculum, 2a edition 2015. 